\title{
Two-Temperature Generalized Thermoelasticity without Energy Dissipation of Infinite Medium with Spherical Cavity Thermally Excited by Time Exponentially Decaying Laser Pulse
}

\author{
Eman A. N. Al-Lehaibi \\ Mathematics Department, College of Science and Arts-Sharoura, Najran University, Najran, KSA \\ Email:sa_1993@hotmail.com
}

Received 14 December 2015; accepted 24 January 2016; published 27 January 2016

Copyright (@ 2015 by author and Scientific Research Publishing Inc.

This work is licensed under the Creative Commons Attribution International License (CC BY).

http://creativecommons.org/licenses/by/4.0/

(c) (i) Open Access

\begin{abstract}
This work is dealing with two-temperature generalized thermoelasticity without energy dissipation infinite medium with spherical cavity when the surface of this cavity is subjected to laser heating pulse. The closed form solutions for the two types of temperature, strain, and the stress distribution due to time exponentially decaying laser pulse are constructed. The Laplace transformation method is employed when deriving the governing equations. The inversion of Laplace transform will be obtained numerically by using the Riemann-sum approximation method. The results have been presented in figures to show the effect of the time exponentially decaying laser pulse and the two temperature parameter on all the studied fields.
\end{abstract}

\section{Keywords}

Generalized Thermoelasticity, Two-Temperature, Energy Dissipation, Laser Pulse

\section{Introduction}

The two temperatures theory of thermoelasticity was introduced by Gurtin and Williams [1], Chen and Gurtin [2], and Chen et al. [3], [4], in which the classical Clausius-Duhem inequality was replaced by another one depending on two temperatures; the conductive temperature $\varphi$ and the thermodynamic temperature $T$, the first is due to the thermal processes, and the second is due to the mechanical processes inherent between the 
particles and the layers of elastic material, this theory was also investigated by Ieşan [5].

Among the authors who contribute to developing this theory, Quintanilla studied existence, structural stability, convergence and spatial behavior for this theory [6], Youssef constructed the generalized Fourier's law to the two-temperature theory of thermoelasticity and proved its uniqueness of solution for homogeneous isotropic material [7]. Puri and Jordan studied the propagation of plane harmonicwaves, recently [8], Magaña and Quintanilla [9] have studied the uniqueness and growth solutions for the model proposed by Youssef [7]. A new theory of generalized thermoelasticity has been constructed based on two-temperature generalized thermoelasticity theory for anisotropic and homogeneous body without energy dissipation by Youssef [10]. This new theorem has been constructed in the context of Green and Naghdi model of type II of linear thermoelasticity. Also, a theorem of general uniqueness is proved for two-temperature generalized thermoelasticity without energy dissipation [10].

The present paper is devoted to a study of the induced temperature and stress fields in aninfinite elastic medium with aspherical cavity under the purview of two-temperature thermoelasticity without energy dissipation. The medium is considered to be an isotropic homogeneous thermoelastic material. The bounding plane surface of the cavity is thermally loaded bytime exponentially decaying laser pulse. An exact solutions of the problem is obtained in Laplace transformdomain, and the inversions of the Laplace transforms have been culculated numerically. The derived formulations are computed numerically for copper, and the results are presented in graphical form.

\section{The Governing Equations}

We will consider perfectly conducting, elastic, isotropic, and homogeneous medium and the governing equations will be taken in the context of two-temperature generalized thermoelasticity without energy dissipation.

According to Youssef model, the heat conduction equation takes the form [10]:

$$
K^{*} \varphi_{, i i}=\rho C_{E} \ddot{T}+\gamma T_{0} \ddot{e}_{k k}-\rho Q, \quad i, k=1,2,3
$$

The conduction-dynamical heat equation takes the form [10]:

$$
\varphi-T=a \varphi_{i i}
$$

The equations of motion take the form

$$
\sigma_{i j},_{j}=\rho \ddot{u}_{i}, i, j=1,2,3
$$

The constitutive equations take the form

$$
\sigma_{i j}=2 \mu e_{i j}+\lambda e_{k k} \delta_{i j}-\gamma\left(T-T_{0}\right) \delta_{i j}
$$

and

$$
e_{i j}=\frac{1}{2}\left(u_{i, j}+u_{j, i}\right)
$$

where $\lambda, \mu$ Lame's constants, $\rho$ density, $C_{E}$ specific heat at constant strain, $\lambda=(3 \lambda+2 \mu) \alpha_{T}, \alpha_{T}$ coefficient of linear thermal expansion, $t$ is the time, $T$ is the temperature, $T_{0}$ is the reference temperature, $\theta=\left(T-T_{0}\right)$ is the thermodynamical temperature increment such that $\frac{|\theta|}{T_{0}} \ll 1, \varphi$ is the conductive heat temperature, $\sigma_{i j}, i, j=r, \psi, z$ are the components of stress tensor, $e$ is the cubic dilatation, $u_{i}$ is the displacement components, $K^{*}$ is the characteristic of Green-Naghdi theorem, a is non-negative parameter (twotemperature parameter), and $\mathrm{Q}$ is the heat source per unit mass.

\section{Formulation of the Problem}

We will consider perfectly conducting elastic infinite bodies with spherical cavity occupy the region $R \leq r<\infty$ of an isotropic homogeneous medium whose state can be written in terms of the space variable $r$ and the time variable $t$ such that all of the field functions vanish at infinity. We use a spherical system of coordinates $(r, \phi, \psi)$. Due to its symmetric geometry, the problem is one-dimensional with all the functions considered 
depending on the radial distance $r$ and the time $t$ and the displacement $u=(u(r), 0,0)$. It is assumed that there are nobody forces in the medium and it is initially quiescent.

Thus, the field equations in spherical one-dimensional case can be put as:

$$
(\lambda+2 \mu) \frac{\partial e}{\partial r}-\gamma \frac{\partial \theta}{\partial r}=\rho \frac{\partial^{2} u}{\partial t^{2}}
$$

and

$$
e=e_{r r}+e_{\phi \phi}+e_{\psi \psi}=e_{r r}
$$

The non-Fourier heat transfer equation due to a laser heating pulse decaying exponentially in time can be written as [7]:

$$
\nabla^{2} \varphi=\frac{\rho C_{E}}{K^{*}} \frac{\partial^{2} \theta}{\partial t^{2}}+\frac{\gamma T_{0}}{K^{*}} \frac{\partial^{2} e}{\partial t^{2}}-\frac{\rho I_{1} \delta}{K^{*}} \mathrm{e}^{-\nu t}
$$

where $I_{1}=\left(1-r_{f}\right) I_{0}$ is the power intensity of surface reflection, $I_{0}$ is laser peak power intensity, $r_{f}$ is reflection coefficient, $v$ laser pulse parameter and $\delta$ is absorption coefficient.

And

$$
\varphi-\theta=a \nabla^{2} \varphi
$$

where $\nabla^{2}=\frac{1}{r^{2}} \frac{\partial}{\partial r}\left(r^{2} \frac{\partial}{\partial r}\right)$.

The constitutive equations will take the following forms

$$
\begin{gathered}
\sigma_{r r}=2 \mu \frac{\partial u}{\partial r}+\lambda e-\gamma \theta \\
\sigma_{\psi \psi}=\sigma_{\varphi \varphi}=2 \mu \frac{u}{r}+\lambda e-\gamma \theta
\end{gathered}
$$

and

$$
\sigma_{r \varphi}=\sigma_{r \psi}=\sigma_{\varphi \psi}=0
$$

where

$$
e=\frac{1}{r^{2}} \frac{\partial\left(r^{2} u\right)}{\partial r}
$$

We shall use the following non-dimensional variablesfor convenience [10]:

$$
\left(r^{\prime}, u^{\prime}, R^{\prime}, \delta^{\prime-1}\right)=c_{o} \eta\left(r, u, R, \delta^{-1}\right), \quad\left(t^{\prime}, v^{\prime-1}\right)=c_{o}^{2} \eta\left(t, v^{-1}\right), \quad \theta^{\prime}=\frac{T-T_{0}}{T_{0}}, \quad \varphi^{\prime}=\frac{\varphi-T_{0}}{T_{0}}, \quad \sigma^{\prime}=\frac{\sigma}{\lambda+2 \mu}
$$

where $c_{o}^{2}=\frac{\lambda+2 \mu}{\rho}$ and $\eta=\frac{\rho C_{E}}{K^{*}}$.

Equation (1) and Equations (4)-(9) assume the form (where the primes are suppressed for simplicity)

$$
\frac{\partial e}{\partial r}-b \frac{\partial \theta}{\partial r}=\frac{\partial^{2} u}{\partial t^{2}}
$$

By using Equation (13) into Equation (15), we get

$$
\nabla^{2} e-b \nabla^{2} \theta=\frac{\partial^{2} e}{\partial t^{2}}
$$

also, we have

$$
\nabla^{2} \varphi=\varepsilon_{1} \frac{\partial^{2} \theta}{\partial t^{2}}+\varepsilon_{2} \frac{\partial^{2} e}{\partial t^{2}}-\varepsilon_{3} \mathrm{e}^{-\nu t}
$$




$$
\begin{gathered}
\varphi-\theta=\varepsilon_{4} \nabla^{2} \varphi \\
\sigma_{r r}=\beta^{2} e-4 \frac{u}{r}-\alpha \theta
\end{gathered}
$$

and

$$
\sigma_{\psi \psi}=\sigma_{\varphi \varphi}=\left(\beta^{2}-2\right) e+2 \frac{u}{r}-\alpha \theta
$$

where $\alpha=\frac{\gamma T_{0}}{\mu}, \quad \varepsilon_{1}=\eta c_{o}^{2}, \quad \varepsilon_{2}=\frac{\gamma c_{0}^{2}}{K^{*}}, \quad \varepsilon_{3}=\frac{\rho \delta I_{1}}{c_{o} T_{0} K^{*} \eta}, \quad \varepsilon_{4}=a c_{o}^{2} \eta^{2}, \quad \beta=\left(\frac{\lambda+2 \mu}{\mu}\right)^{1 / 2}$ and $b=\frac{\alpha}{\beta^{2}}$.

\section{The Solution in the Laplace Transform Domain}

We use the Laplace transform of both sides of the last equations defined as:

$$
\bar{f}(s)=\int_{0}^{\infty} f(t) \mathrm{e}^{-s t} \mathrm{~d} t
$$

Hence, we obtain

$$
\begin{gathered}
\nabla^{2} \bar{e}-b \nabla^{2} \bar{\theta}=s^{2} \bar{e} \\
\nabla^{2} \bar{\varphi}=\varepsilon_{1} s^{2} \bar{\theta}+\varepsilon_{2} s^{2} \bar{e}-\frac{\varepsilon_{3}}{s+v} \\
\bar{\theta}=\bar{\varphi}-\varepsilon_{4} \nabla^{2} \bar{\varphi} \\
\bar{\sigma}_{r r}=\beta^{2} \bar{e}-4 \frac{\bar{u}}{r}-\alpha \bar{\theta} \\
\bar{\sigma}_{\psi \psi}=\bar{\sigma}_{\varphi \varphi}=\left(\beta^{2}-2\right) \bar{e}+2 \frac{\bar{u}}{r}-\alpha \bar{\theta}
\end{gathered}
$$

and

$$
\bar{e}=\frac{1}{r^{2}} \frac{\partial\left(r^{2} \bar{u}\right)}{\partial r}
$$

Eliminating $\bar{\theta}$ between Equations (23) and (24), we have

$$
\nabla^{2} \bar{\varphi}=\alpha_{1} \bar{\varphi}+\alpha_{2} \bar{e}-\alpha_{3}
$$

where $\alpha_{1}=\frac{\varepsilon_{1} s^{2}}{1+\varepsilon_{1} \varepsilon_{4} s^{2}}, \quad \alpha_{2}=\frac{\varepsilon_{2} s^{2}}{1+\varepsilon_{1} \varepsilon_{4} s^{2}}$ and $\alpha_{3}=\frac{\varepsilon_{3}}{\left(1+\varepsilon_{1} \varepsilon_{4} s^{2}\right)(s+v)}$.

From Equations (28) and (24), we obtain

$$
\bar{\theta}=\left(1-\varepsilon_{4} \alpha_{1}\right) \bar{\varphi}-\varepsilon_{4} \alpha_{2} \bar{e}+\varepsilon_{4} \alpha_{3}
$$

From Equations (22) and (28), we have

$$
\nabla^{2} \bar{e}=\beta_{1} \bar{\varphi}+\beta_{2} \bar{e}-\beta_{3}
$$

where $\beta_{1}=\frac{\alpha_{1}\left(1-\varepsilon_{4} \alpha_{1}\right) b}{\left(1+\varepsilon_{4} \alpha_{2} b\right)}, \quad \beta_{2}=\frac{\left(\alpha_{2}\left(1-\varepsilon_{4} \alpha_{1}\right) b+s^{2}\right)}{\left(1+\varepsilon_{4} \alpha_{2} b\right)}$ and $\beta_{3}=\frac{\alpha_{3}\left(1-\varepsilon_{4} \alpha_{1}\right) b}{\left(1+\varepsilon_{4} \alpha_{2} b\right)}$.

By eliminating $\bar{e}$ between Equations (28) and (30), we obtain

$$
\left[\nabla^{4}-L \nabla^{2}+M\right] \bar{\varphi}=N_{1}
$$

where $L=\alpha_{1}+\beta_{2}, \quad M=\alpha_{1} \beta_{2}-\alpha_{2} \beta_{1}$ and $N_{1}=\beta_{2} \alpha_{3}-\alpha_{2} \beta_{3}$. 
By eliminating $\bar{\varphi}$ between Equations (28) and (30), we get

$$
\left[\nabla^{4}-L \nabla^{2}+M\right] \bar{e}=N_{2}
$$

where $N_{2}=\alpha_{1} \beta_{3}-\alpha_{3} \beta_{1}$.

The bounded solutions of the Equations (31) and (32) take the forms

$$
\bar{\varphi}=\frac{N_{1}}{M}+\sum_{i=1}^{2} A_{i} \frac{\mathrm{e}^{-p_{i} r}}{r}
$$

and

$$
\bar{e}=\frac{N_{2}}{M}+\sum_{i=1}^{2} B_{i} \frac{\mathrm{e}^{-p_{i} r}}{r}
$$

where $\pm p_{1}, \pm p_{2}$ are the roots of the equation

$$
p^{4}-L p^{2}+M=0
$$

By using Equations (33) and (34) into Equation (30), we obtain

$$
A_{i}=\frac{B_{i}\left(p_{i}^{2}-\beta_{2}\right)}{\beta_{1}}
$$

Hence, we get

$$
\bar{\varphi}=\frac{N_{1}}{M}+\frac{1}{\beta_{1}} \sum_{i=1}^{2} B_{i}\left(p_{i}^{2}-\beta_{2}\right) \frac{\mathrm{e}^{-p_{i} r}}{r}
$$

To get the constants $B_{1}$ and $B_{2}$, we have to apply the boundary conditions on the surface of the cavity when $r=R$ ( $R$ is the radius of the cavity). We will consider that the heat conduction $\bar{\varphi}$ and the strain $\bar{e}$ have zero value on the surface of the cavity, which gives that

$$
\bar{\varphi}(R, s)=\bar{e}(R, s)=0
$$

Thus, the system of the equations on (34) and (37) gives the following linear equations

$$
\sum_{i=1}^{2} B_{i} \mathrm{e}^{-p_{i} R}=-\frac{N_{2} R}{M}
$$

and

$$
\sum_{i=1}^{2} B_{i}\left(p_{i}^{2}-\beta_{2}\right) \mathrm{e}^{-p_{i} R}=-\frac{\beta_{1} N_{1} R}{M}
$$

By solving the above system, we get

$$
B_{1}=\frac{\operatorname{Re}^{p_{1} R}\left[N_{1} \beta_{1}-N_{2}\left(p_{2}^{2}-\beta_{2}\right)\right]}{M\left(p_{2}^{2}-p_{1}^{2}\right)} \text { and } B_{2}=-\frac{R^{p_{2} R}\left[N_{1} \beta_{1}-N_{2}\left(p_{1}^{2}-\beta_{2}\right)\right]}{M\left(p_{2}^{2}-p_{1}^{2}\right)}
$$

Those complete the solutions as following

$$
\begin{aligned}
\bar{\varphi}(s, r)= & \frac{N_{1}}{M}+\left(\frac{R\left[N_{1} \beta_{1}-N_{2}\left(p_{2}^{2}-\beta_{2}\right)\right]\left(p_{1}^{2}-\beta_{2}\right)}{\beta_{1} M\left(p_{2}^{2}-p_{1}^{2}\right)}\right) \frac{\mathrm{e}^{-p_{1}(r-R)}}{r} \\
& -\left(\frac{R\left[N_{1} \beta_{1}-N_{2}\left(p_{1}^{2}-\beta_{2}\right)\right]\left(p_{2}^{2}-\beta_{2}\right)}{\beta_{1} M\left(p_{2}^{2}-p_{1}^{2}\right)}\right) \frac{\mathrm{e}^{-p_{2}(r-R)}}{r}
\end{aligned}
$$




$$
\bar{e}(s, r)=\frac{N_{2}}{M}+\left(\frac{R\left[N_{1} \beta_{1}-N_{2}\left(p_{2}^{2}-\beta_{2}\right)\right]}{M\left(p_{2}^{2}-p_{1}^{2}\right)}\right) \frac{\mathrm{e}^{-p_{1}(r-R)}}{r}-\left(\frac{R\left[N_{1} \beta_{1}-N_{2}\left(p_{1}^{2}-\beta_{2}\right)\right]}{M\left(p_{2}^{2}-p_{1}^{2}\right)}\right) \frac{\mathrm{e}^{-p_{2}(r-R)}}{r}
$$

Substituting from Equations (41) and (42) in (28), (27) and (25) we get $\bar{\theta}(s, r), \bar{\sigma}(s, r)$ and $\bar{u}(s, r)$ respectively.

\section{Numerical Inversion of the Laplace Transform}

To determine the solutions in the time domain, the Riemann-sum approximation method is used to obtain the numerical results. In this method, any function in Laplace domain can be inverted to the time domain as:

$$
f(t)=\frac{\mathrm{e}^{\kappa t}}{t}\left[\frac{1}{2} \bar{f}(\kappa)+\operatorname{Re} \sum_{n=1}^{N}(-1)^{n} \bar{f}\left(\kappa+\frac{i n \pi}{t}\right)\right]
$$

where Re is the real part and $i$ is imaginary number unit. For faster convergence, numerous numerical experiments have shown that the value of $\kappa$ satisfies the relation $\kappa t \approx 4.7$ Tzou [9].

\section{Numerical Results and Discussion}

We now consider a numerical example for which computational results are given. For this purpose, copper is taken as the thermoelastic material for which we take the following values of the different physical constants [11]:

$$
\begin{gathered}
K=386 \mathrm{~kg} \cdot \mathrm{m} \cdot \mathrm{K}^{-1} \cdot \mathrm{s}^{-3}, \quad \alpha_{T}=1.78 \times(10)^{-5} \mathrm{~K}^{-1}, \quad \rho=8954 \mathrm{~kg} \cdot \mathrm{m}^{-3}, \quad C_{E}=383.1 \mathrm{~m}^{2} \cdot \mathrm{K}^{-1} \cdot \mathrm{s}^{-2}, \\
T_{0}=293 \mathrm{~K}, \quad \mu=3.86 \times(10)^{10} \mathrm{~kg} \cdot \mathrm{m}^{-1} \cdot \mathrm{s}^{-2}, \quad \lambda=7.76 \times(10)^{10} \mathrm{~kg} \cdot \mathrm{m}^{-1} \cdot \mathrm{s}^{-2}, \quad \beta^{2}=4, \quad R=1.0, \quad t=0.2
\end{gathered}
$$

From the above values, we get the non-dimensional values of the problem as:

$$
b=0.010444, \alpha=0.000143, \varepsilon_{1}=7.98, \varepsilon_{2}=12.836, \varepsilon_{3}=2.14 .
$$

Figures 1-5 represent the conductive temperature distribution, the thermodynamic temperature distribution, the strain distribution, the displacement distribution, and the stress distribution respectively, in the context of one-temperature type (solid lines) and two-temperature type (dashed lines). We can notice that the two-temperature parameter has significant effects on all distribution. The material reaches the steady state through the twotemperature type before the one-temperature type. The peak points decrease when we use the two-temperature type.

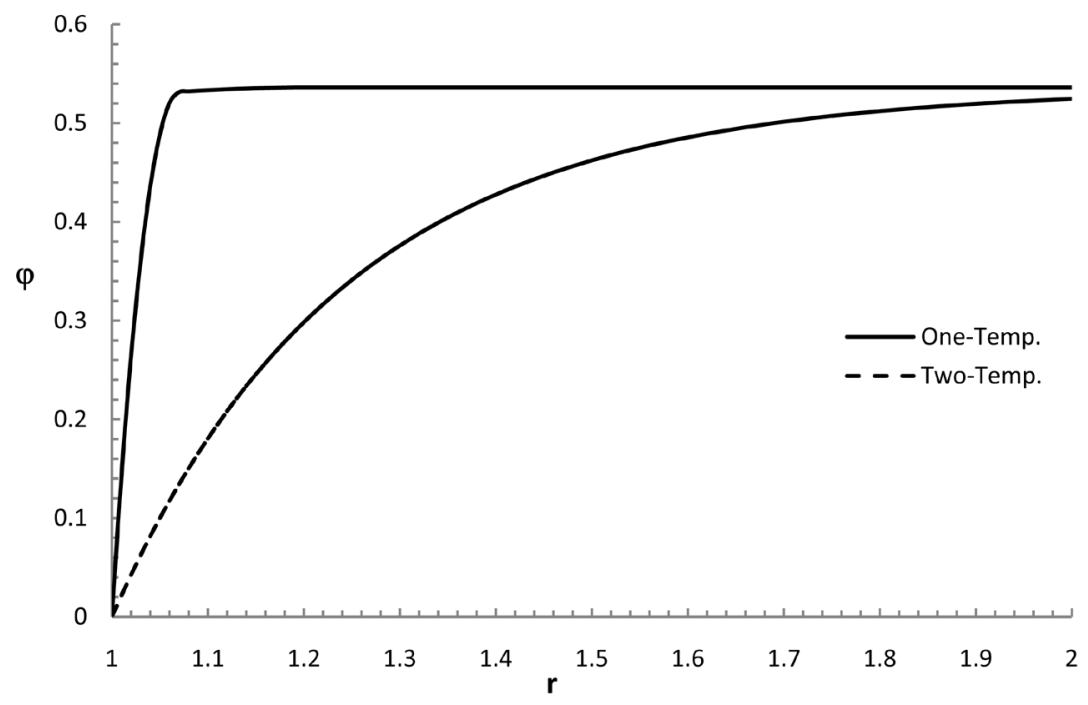

Figure 1. The conductive temperature distribution. 


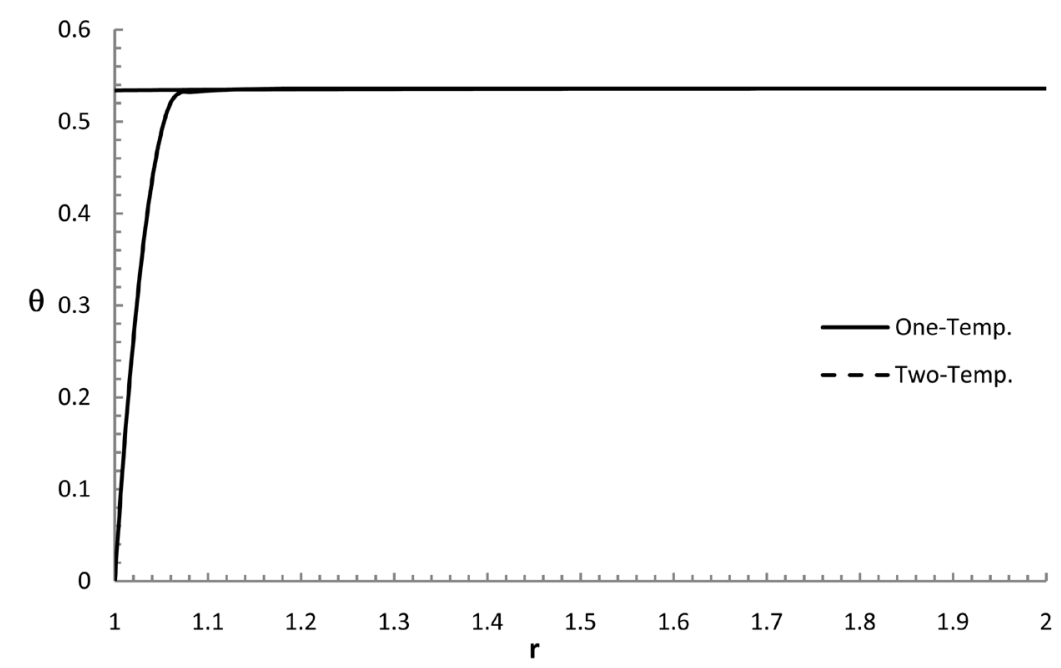

Figure 2. The thermodynamic temperature distribution.

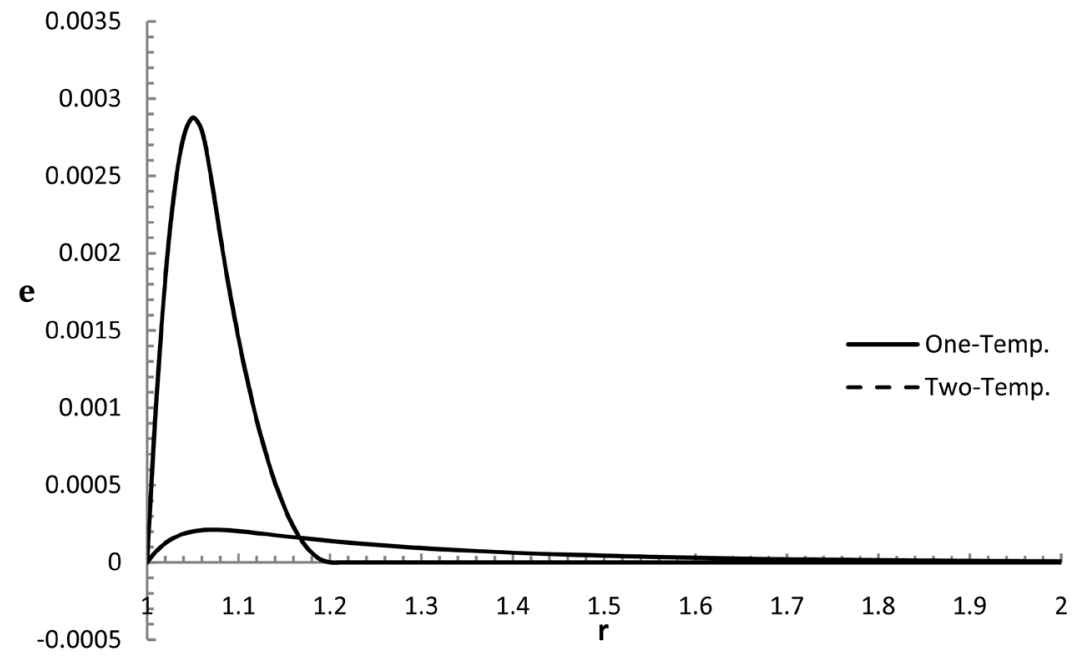

Figure 3. The strain distribution.

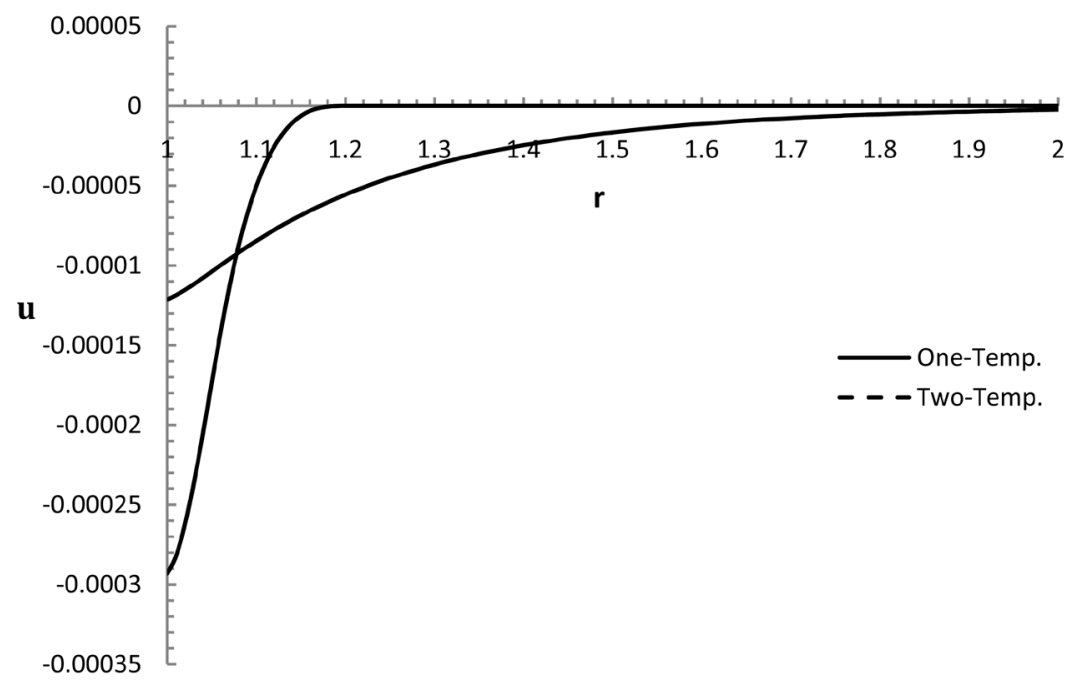

Figure 4. The displacement distribution. 


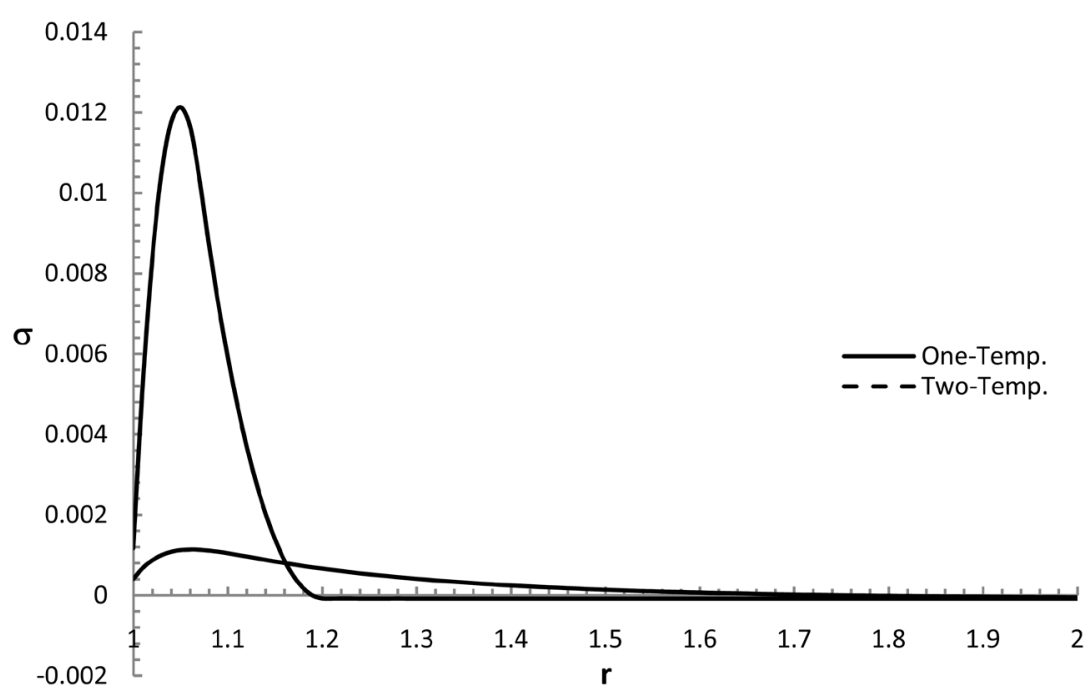

Figure 5. The stress distribution.

\section{Acknowledgements}

I want thank Prof. Hamdy M. Youssef (Mechanics Department, Faculty of Engineering, Umm Al-Qura University, Makkah KSA) for his help and advises to me to complete this work and to choose this respected journal.

\section{References}

[1] Gurtin, M.E. and Williams, W.O. (1967) An Axiomatic Foundation for Continuum Thermodynamics. Archive for Rational Mechanics and Analysis, 26, 83-117. http://dx.doi.org/10.1007/BF00285676

[2] Chen, P.J. and Gurtin, M.E. (1968) On a Theory of Heat Conduction Involving Two Temperatures. Zeitschrift für Angewandte Mathematik und Physik (ZAMP), 19, 614-627. http://dx.doi.org/10.1007/BF01594969

[3] Chen, P.J., Gurtin, M.E. and Williams, W.O. (1969) On the Thermodynamics of Non-Simple Elastic Materials with Two Temperatures. Zeitschrift für Angewandte Mathematik und Physik (ZAMP), 20, 107-112. http://dx.doi.org/10.1007/BF01591120

[4] Chen, P.J. and Williams, W.O. (1968) A Note on Non-Simple Heat Conduction. Zeitschrift für Angewandte Mathematik und Physik (ZAMP), 19, 969-970. http://dx.doi.org/10.1007/BF01602278

[5] Ieşan, D. (1970) On the Linear Coupled Thermoelasticity with Two Temperatures. Zeitschrift für Angewandte Mathematik und Physik (ZAMP), 21, 583-591. http://dx.doi.org/10.1007/BF01587687

[6] Quintanilla, R. (2004) On Existence, Structural Stability, Convergence and Spatial Behavior in Thermoelasticity with Two Temperatures. Acta Mechanica, 168, 61-73. http://dx.doi.org/10.1007/s00707-004-0073-6

[7] Youssef, H.M. (2006) Theory of Two-Temperature-Generalized Thermoelasticity. IMA Journal of Applied Mathematics, 71, 383-90. http://dx.doi.org/10.1093/imamat/hxh101

[8] Puri, P. and Jordan, P.M. (2006) On the Propagation of Harmonic Plane Waves under the Two-Temperature Theory. International Journal of Engineering Science, 44, 1113-1126. http://dx.doi.org/10.1016/j.ijengsci.2006.07.002

[9] Magaña, A. and Quintanilla, R. (2009) Uniqueness and Growth of Solutions in Two-Temperature Generalized Thermoelastic Theories. Mathematics and Mechanics of Solids, 14, 622-634. http://dx.doi.org/10.1177/1081286507087653

[10] Youssef, H.M. (2011) Theory of Two-Temperature Thermoelasticity without Energy Dissipation. Journal of Thermal Stresses, 34, 138-146. http://dx.doi.org/10.1080/01495739.2010.511941

[11] Youssef, H.M. and Al-Harby, A.H. (2007) State-Space Approach of Two-Temperature Generalized Thermoelasticity of Infinite Body with a Spherical Cavity Subjected to Different Types of Thermal Loading. Archive of Applied Mechanics, 77, 675-687. http://dx.doi.org/10.1007/s00419-007-0120-6 